\title{
On the efficient evaluation of hyper-singular integrals in Galerkin surface integral equation formulations via the direct evaluation method
}

\author{
J.M. Tamayo*(1) ${ }^{(1)}$ A.G. Polimeridis ${ }^{(2)}$, J.M. Rius $^{(1)}$, and J.R. Mosig $^{(2)}$ \\ (1) Dept. of Signal Theory \& Comm, Polythecnic University of Catalonia, \\ c/ J. Girona 1, 08034, Barcelona, Spain. \\ ${ }^{(2)}$ Lab. of Electromagnetics and Acoustics (LEMA), Ecole Polytechnique \\ Fédérale de Lausanne (EPFL), CH-1015 Lausanne, Switzerland. \\ E-mail: jose.maria.tamayo@tsc.upc.edu
}

\section{Introduction}

Surface integral equation formulations are proven to be one of the most powerful methods for the solution of various electromagnetic (EM) antenna and scattering problems. Galerkin variants of the method of moments (MoM) are most often utilized for the numerical solution of these electromagnetic surface integral equations, calling for the calculation of fourdimensional integrals with singular kernels. Due to the non smooth behavior of the singular integral kernels, classical numerical quadrature rules fail to meet the requirements for high precision results, and more sophisticated techniques are needed.

To be more specific, weakly singular integrals appear in the electric field integral equation (EFIE) formulations, when divergence-conforming basis and testing functions, like the popular Rao-Wilton-Glisson (RWG), are incorporated in the Galerkin MoM. On the other hand, 4-D hyper-singular integrals (following here and for the rest of the manuscript the convention used in EM community) arise in the numerical solution of magnetic field integral equation (MFIE) and combined field integral equation (CFIE) formulations, since the latter are combination of EFIE and MFIE formulations. Over the last years, numerous techniques have been presented for the accurate and efficient evaluation of those multidimensional hyper-singular integrals. They can be roughly categorized into two main families: singularity cancellation methods [1], in which our method could be included, and singularity subtraction methods $[2,3,4]$. Although both methods seem to lead to superior accuracy compared to direct implementation of typical quadrature rules, to the best of our knowledge, the quest for machine precision results, together with improved efficiency, remains still unfinished.

In this paper, the direct evaluation method tailored for the hyper-singular integrals arising in Galerkin surface integral equation formulations is developed. The highly abbreviated derivation which follows will be duly expanded at the conference. The proposed method utilizes a series of coordinate transformations together with a re-ordering of the integrations in order to reduce the 4-D hyper-singular integrals to 2-D smooth integrals that can be easily computed via generalized Cartesian product rules based on standard Gauss quadratures, readily available in the literature. The direct evaluation method was originally introduced by Gray et al. [5, 6] for the evaluation of super hyper-singular Galerkin surface integrals

\footnotetext{
${ }^{0}$ This work was supported partially by the Spanish Interministerial Commission on Science and Technology (CICYT) under projects TEC2006-13248-C04-02/TCM and TEC2007-66698-C04-01/TCM and CONSOLIDER CSD2008-00068 and by the Ministerio de Educación y Ciencia through the FPU fellowship program.
} 
arising in static problems and recently it was extended for the case of the weakly singular integrals in Galerkin MoM problems over coincident triangle elements [7].

\section{Direct evaluation method}

We are going to deal with the evaluation of the following hyper-singular integral arising in either the MFIE or the CFIE formulations, when the triangles $E_{P}$ and $E_{Q}$ from the discretization have a common edge (see Fig. 1):

$$
I:=\int_{E_{P}} \mathbf{g}(\mathbf{r}) \cdot \int_{E_{Q}} \nabla G\left(\mathbf{r}, \mathbf{r}^{\prime}\right) \times \mathbf{f}\left(\mathbf{r}^{\prime}\right) d S^{\prime} d S
$$

being $\mathbf{f}\left(\mathbf{r}^{\prime}\right)$ a RWG basis function whose support includes the triangle $E_{Q}$ and $\mathbf{g}(\mathbf{r})$ a testing function which can be a RWG or a $\hat{\mathbf{n}} \times$ RWG function defined over $E_{P} . G\left(\mathbf{r}, \mathbf{r}^{\prime}\right)$ corresponds to the free space Green's function, depending on the distance $R=\left|\mathbf{r}-\mathbf{r}^{\prime}\right|, G(R)=$ $e^{-j k R} / R$.

The following set of variable transformations is held to completely cancel out the singularity $1 / R^{2}$ over the common edge. First, we move to equilateral parametric spaces $\{\eta, \xi\}$ and $\left\{\eta^{\prime}, \xi^{\prime}\right\}$ in each triangle (see Fig. 1), following the original work by Gray et al. [5, 6]. Afterwards, we perform two consecutive polar coordinate changes

$$
\begin{aligned}
& \eta^{\prime}=\rho \cos (\theta)-\eta \quad \text { and } \begin{aligned}
\rho & =\Lambda \cos (\Psi) \\
\xi^{\prime} & =\rho \sin (\theta)
\end{aligned} \quad \begin{array}{l}
\xi \\
\end{array} \quad \Lambda \sin (\Psi)
\end{aligned}
$$

obtaining finally that the aimed integral (1) can be expressed in terms of the new parameters as

$$
\begin{aligned}
I= & \int_{0}^{1} d \eta \int_{0}^{\Theta_{1}} d \theta \int_{0}^{\Psi_{C}} d \Psi \int_{0}^{\Lambda_{L_{3}}} F d \Lambda+\int_{0}^{1} d \eta \int_{0}^{\Theta_{1}} d \theta \int_{\Psi_{C}}^{\pi / 2} d \Psi \int_{0}^{\Lambda_{L_{2}}} F d \Lambda \\
& +\int_{0}^{1} d \eta \int_{\Theta_{1}}^{\pi} d \theta \int_{0}^{\Psi_{A}} d \Psi \int_{0}^{\Lambda_{L_{1}}} F d \Lambda+\int_{0}^{1} d \eta \int_{\Theta_{1}}^{\pi} d \theta \int_{\Psi_{A}}^{\pi / 2} d \Psi \int_{0}^{\Lambda_{L_{2}}} F d \Lambda
\end{aligned}
$$

where the integrand function $F(\Lambda, \eta, \Psi, \theta)$ equals

$$
\begin{aligned}
F(\Lambda, \eta, \Psi, \theta)= & F^{+}(\Lambda, \eta, \Psi, \theta)+F^{+}(\Lambda,-\eta, \Psi, \pi-\theta) \\
F^{+}(\Lambda, \eta, \Psi, \theta)= & \frac{A_{p} A_{q}}{3} \cos (\Psi)\left(\frac{e^{-j k \Lambda B(\theta, \Psi)}}{B(\theta, \Psi)^{3}}(1+j k \Lambda B(\theta, \Psi))\right) \\
& \mathbf{g}(\Lambda, \eta, \theta, \Psi) \cdot(\mathbf{f}(\Lambda, \eta, \theta, \Psi) \times \mathbf{B}(\theta, \Psi))
\end{aligned}
$$

and the expressions of the integration limits are omitted for the sake of brevity. Last expression uses the key fact that after the transformations the distance vector $\mathbf{R}=\mathbf{r}-\mathbf{r}^{\prime}$ takes the form $\mathbf{R}=\Lambda \mathbf{B}(\theta, \Psi)$ and the $\Lambda=0$ singularity is gone due to the Jacobian of the transformation

$$
J=\frac{A_{p} A_{q}}{3} \Lambda^{2} \cos (\Psi) .
$$

No matter which actual $\mathbf{g}$ and $\mathbf{f}$ (RWG or $\hat{\mathbf{n}} \times \mathrm{RWG}$ ) basis functions are used, it is provable that the integrand $F$ is analytically integrable with respect to $\Lambda$. Furthermore, the result is in turn analytically integrable in terms of $\eta$. Therefore, it is interesting to suitably change the order of the integrals in (3) placing the integration with respect to $\eta$ right after the integration with respect to $\Lambda$. After this procedure the integral (1) takes the form:

$$
\begin{aligned}
I & =\int_{0}^{\pi / 3} d \theta \int_{0}^{\Psi_{B}} \chi^{a}(\theta, \Psi) d \Psi+\int_{\pi / 3}^{\pi / 2} d \theta \int_{\Psi_{A}}^{\Psi_{B}} \chi^{a}(\theta, \Psi) d \Psi+\int_{\pi / 3}^{\pi / 2} d \theta \int_{0}^{\Psi_{A}} \chi^{b}(\theta, \Psi) d \Psi \\
& +\int_{\pi / 2}^{\pi} d \theta \int_{0}^{\Psi_{A}} \chi^{c}(\theta, \Psi) d \Psi+\int_{0}^{\pi / 2} d \theta \int_{\Psi_{B}}^{\pi / 2} \chi^{d}(\theta, \Psi) d \Psi+\int_{\pi / 2}^{\pi} d \theta \int_{\Psi_{A}}^{\pi / 2} \chi^{d}(\theta, \Psi) d \Psi
\end{aligned}
$$


being $\chi^{\alpha}(\theta, \Psi)$ with $\alpha \in\{a, b, c, d\}$ analytic 2-D sufficiently smooth functions depending on the angle parameters $\theta$ and $\Psi$. These functions will be presented in a very compact and ready-to-use form so that the direct evaluation method finds its place in standard mathematical subroutine libraries widely used in the computational electromagnetics community.

\section{Numerical results}

A study on the accuracy and efficiency of the proposed method is shown in this section. Taking into account the short influence of the basis and testing functions $\mathbf{f}$ and $\mathbf{g}$, it suffices to analyze among the various combinations of functions a single, albeit representative, case. In particular, following the notation in Fig. 1, we choose the case $\mathbf{f}\left(\mathbf{r}^{\prime}\right)=\mathbf{f}_{1}^{\prime}\left(\mathbf{r}^{\prime}\right)$ and $\mathbf{g}(\mathbf{r})=$ $\mathbf{f}_{3}(\mathbf{r})$ where

$$
\mathbf{f}_{3}(\mathbf{r})=\frac{\left|\mathbf{r}_{2}-\mathbf{r}_{1}\right|}{2 A_{p}}\left(\mathbf{r}-\mathbf{r}_{3}\right) ; \quad \mathbf{f}_{1}^{\prime}\left(\mathbf{r}^{\prime}\right)=\frac{\left|\mathbf{r}_{4}-\mathbf{r}_{2}\right|}{2 A_{q}}\left(\mathbf{r}^{\prime}-\mathbf{r}_{2}\right) .
$$

We consider two edge adjacent triangles with the following vertices:

$$
\mathbf{r}_{1}=[0,0,0] ; \quad \mathbf{r}_{2}=[0,0.1 \lambda, 0] ; \quad \mathbf{r}_{3}=[0,0,0.1 \lambda] ; \quad \mathbf{r}_{4}=[0.1 \lambda, 0,0]
$$

where $\lambda=1[\mathrm{~m}]$ corresponds to the wavelength. Note that $\mathbf{r}_{1}$ and $\mathbf{r}_{2}$ are the two common vertices.

Fig. 2 shows the accuracy and efficiency of the proposed method. It proves the excellent convergence of the method, reaching a relative error smaller than $10^{-5}$ in $10 \mathrm{~ms}$ and almost machine precision in about $100 \mathrm{~ms}$. The used codes have been implemented in MATLAB ${ }^{\circledR}$ and the simulations have been run in a computer with an Intel ${ }^{\circledR}$ Core 2 Duo CPU at $2 \mathrm{GHz}$ (no parallelization has been done), Windows XP and MATLAB ${ }^{\circledR}$ R2007b.

Moreover, the reference solution has been obtained with 2500 integration points using our direct evaluation method including two analytical integrations. In view of the results depicted in Fig. 2, we can infer that the proposed method reaches an exact value up to 14 significant digits and it equals:

$$
I=3.4928883683897 \cdot 10^{-3}-j 2.2540732129690 \cdot 10^{-5} .
$$

The validity of the proposed algorithm has been also corroborated with the help of the singularity subtraction method [4].

\section{Conclusions}

In this paper, the direct evaluation method is outlined for the computation of the hypersingular integrals arising in Galerkin surface integral equation formulations. The key feature of the proposed scheme lies on the appropriate regularization of the singular integrand together with the reduction of the dimensionality of the original integral from 4-D to 2-D. The procedure presented herein succeed in providing numerical results of unmatched accuracy (close to the machine precision) and efficiency, thus, improving substantially the accuracy of the impedance matrix elements in field integral equation formulations as well as reducing the overall filling time. 


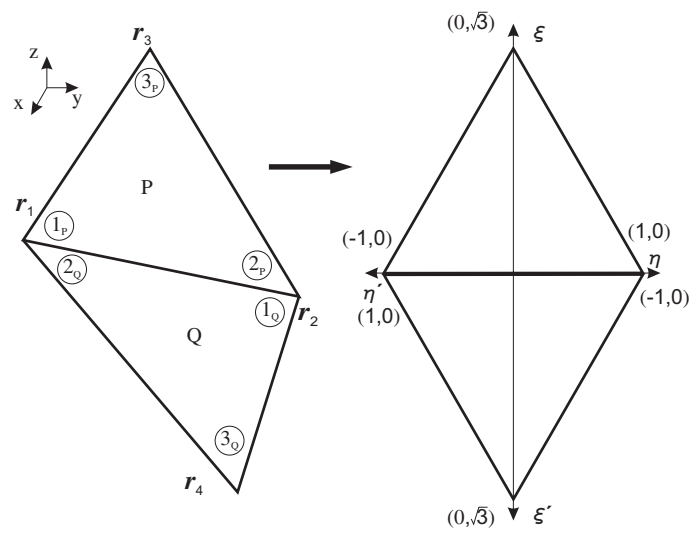

Figure 1: Orientation of the triangular elements both in the original and the equilateral triangle parameter space.

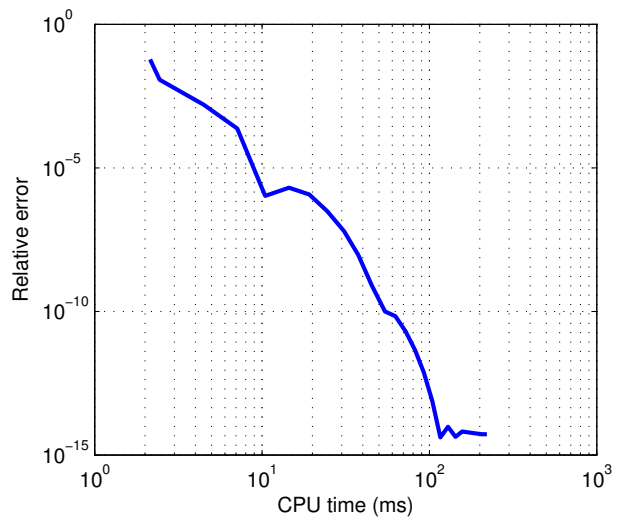

Figure 2: Relative error as a function of the computational time.

\section{References}

[1] P. W. Fink, D. R. Wilton, and M. A. Khayat, "Simple and efficient numerical evaluation of near-hypersingular integrals," IEEE Antennas Wireless Propag. Lett., vol. 7, pp. 469472, 2008.

[2] R. D. Graglia, "On the numerical integration of the linear shape functions times the 3D Green's function or its gradient on a plane triangle," IEEE Trans. Antennas Propag., vol. 41, no. 10, pp. 1448-1455, Oct. 1993.

[3] T. F. Eibert and V. Hansen, "On the calculation of potential integrals for linear source distributions on triangular domains," IEEE Trans. Antennas Propag., vol. 43, no. 12, pp. 1499-1502, Dec. 1995.

[4] P. Yla-Oijala and M. Taskinen, "Calculation of CFIE impedance matrix elements with RWG and $\hat{n} \times$ RWG functions," IEEE Trans. Antennas Propag., vol. 51, no. 8, pp. 1837-1846, Aug. 2003.

[5] L. J. Gray, J. M. Glaeser, and T. Kaplan, "Direct evaluation of hypersingular Galerkin surface integrals," SIAM J. Sci. Comput., vol. 25, no. 5, pp. 1534-1556, 2004.

[6] L. J. Gray, A. Salvadori, A. V. Phan, and A. Mantic, "Direct evaluation of hypersingular Galerkin surface integrals. II," Electronic Journal of Boundary Elements, vol. 4, no. 3, pp. 105-130, 2006.

[7] A. G. Polimeridis and T. V. Yioultsis, "On the direct evaluation of weakly singular integrals in Galerkin mixed potential integral equation formulations," IEEE Trans. Antennas Propag., vol. 56, no. 9, pp. 3011-3019, Sep. 2008. 